\title{
Yoga for chronic low back pain: A meta-analysis of randomized controlled trials
}

\author{
Susan Holtzman PhD, R Thomas Beggs MA
}

\author{
S Holtzman, RT Beggs. Yoga for chronic low back pain: A meta- \\ analysis of randomized controlled trials. Pain Res Manag \\ $2013 ; 18(5): 267-272$.
}

OBJECTIVES: To evaluate the efficacy of yoga as an intervention for chronic low back pain (CLBP) using a meta-analytical approach. Randomized controlled trials (RCTs) that examined pain and/or functional disability as treatment outcomes were included. Post-treatment and follow-up outcomes were assessed.

METHODS: A comprehensive search of relevant electronic databases, from the time of their inception until November 2011, was conducted. Cohen's d effect sizes were calculated and entered in a random-effects model.

RESULTS: Eight RCTs met the criteria for inclusion (eight assessing functional disability and five assessing pain) and involved a total of 743 patients. At post-treatment, yoga had a medium to large effect on functional disability $(\mathrm{d}=0.645)$ and pain $(\mathrm{d}=0.623)$. Despite a wide range of yoga styles and treatment durations, heterogeneity in post-treatment effect sizes was low. Follow-up effect sizes for functional disability and pain were smaller, but remained significant $(\mathrm{d}=0.397$ and $\mathrm{d}=0.486$, respectively); however, there was a moderate to high level of variability in these effect sizes.

DISCUSSION: The results of the present study indicate that yoga may be an efficacious adjunctive treatment for CLBP. The strongest and most consistent evidence emerged for the short-term benefits of yoga on functional disability. However, before any definitive conclusions can be drawn, there are a number of methodological concerns that need to be addressed. In particular, it is recommended that future RCTs include an active control group to determine whether yoga has specific treatment effects and whether yoga offers any advantages over traditional exercise programs and other alternative therapies for CLBP.

Key Words: Chronic low back pain; Meta-analysis; Yoga

\section{Le yoga pour soulager les douleurs lombaires : une méta-analyse d'essais aléatoires et contrôlés}

\begin{abstract}
OBJECTIFS : Les chercheurs ont évalué l'efficacité du yoga comme intervention contre les douleurs lombaires chroniques (DLC) au moyen d'une approche méta-analytique. Ils ont inclus des essais aléatoires et contrôlés (EAC) qui portaient sur la douleur ou l'invalidité fonctionnelle comme issues du traitement. Ils ont évalué les issues après le traitement et au suivi.
\end{abstract}

MÉTHODOLOGIE : Les chercheurs ont procédé à une recherche exhaustive des bases de données virtuelles pertinentes, à compter de leur création jusqu'à novembre 2011. Ils ont calculé les tailles d'effet d de Cohen et les ont saisies dans un modèle à effets aléatoires.

RÉSULTATS : Huit EAC respectaient les critères d'inclusion (huit évaluant l'invalidité fonctionnelle et cinq, la douleur) et portaient sur un total de 743 patients. Après le traitement, le yoga avait un effet moyen à important sur l'invalidité fonctionnelle $(d=0,645)$ et la douleur $(d=0,623)$. Malgré un vaste éventail de styles de yoga et de durées de traitement, l'hétérogénéité des tailles d'effet après le traitement était faible. Les tailles d'effet de suivi étaient plus faibles sur le plan de l'invalidité fonctionnelle et de la douleur, mais demeuraient significatives $(\mathrm{d}=0,397$ et $\mathrm{d}=0,486$, respectivement). Cependant, le taux de variabilité de ces tailles d'effet était modéré à élevé.

EXPOSÉ : Selon les résultats de la présente étude, le yoga peut être un traitement d'appoint efficace des DLC. Les données probantes les plus solides et les plus cohérentes faisaient foi des effets bénéfiques à court terme du yoga sur l'invalidité fonctionnelle. Cependant, avant de pouvoir tirer une conclusion définitive, il faudra régler un certain nombre de problèmes méthodologiques. Notamment, on recommande que les futurs EAC incluent un groupe de contrôle actif pour déterminer si le yoga avait des effets thérapeutiques précis et s'il comportait des avantages par rapport aux programmes d'exercice classiques et aux autres traitements de la DLC.

Yoga is an ancient practice that originated in India $>4000$ years ago and consists of several key components, including physical postures ('asanas'), breathing techniques ('pranayama'), relaxation and meditation ('dhyana') (9). A biopsychosocial approach is now considered to be the gold standard for treating chronic pain (10). Therefore, the holistic approach to healing involved in yoga holds intuitive appeal for the treatment of CLBP. Indeed, there is a small but growing body of literature suggesting that yoga can increase muscular strength, joint flexibility and balance among patients with CLBP (9,11-13). Recent research has also indicated that yoga can reduce pain catastrophizing, increase pain acceptance and improve overall emotional functioning among individuals with chronic pain (14-17). It has also been suggested that the group format of most yoga classes may provide patients with a sense of belonging and increase the availability of emotional and tangible support (18). This is noteworthy because each of these factors are known to be important contributors to pain and pain-related disability among patients with CLBP (19).

Randomized controlled trials (RCTs) are increasingly being used to evaluate the efficacy of yoga for CLBP. In a recent narrative systematic review of the literature, Posadzki et al (20) identified seven RCTs that have been conducted on yoga for CLBP, and noted that yoga led to a including chronic pain. 


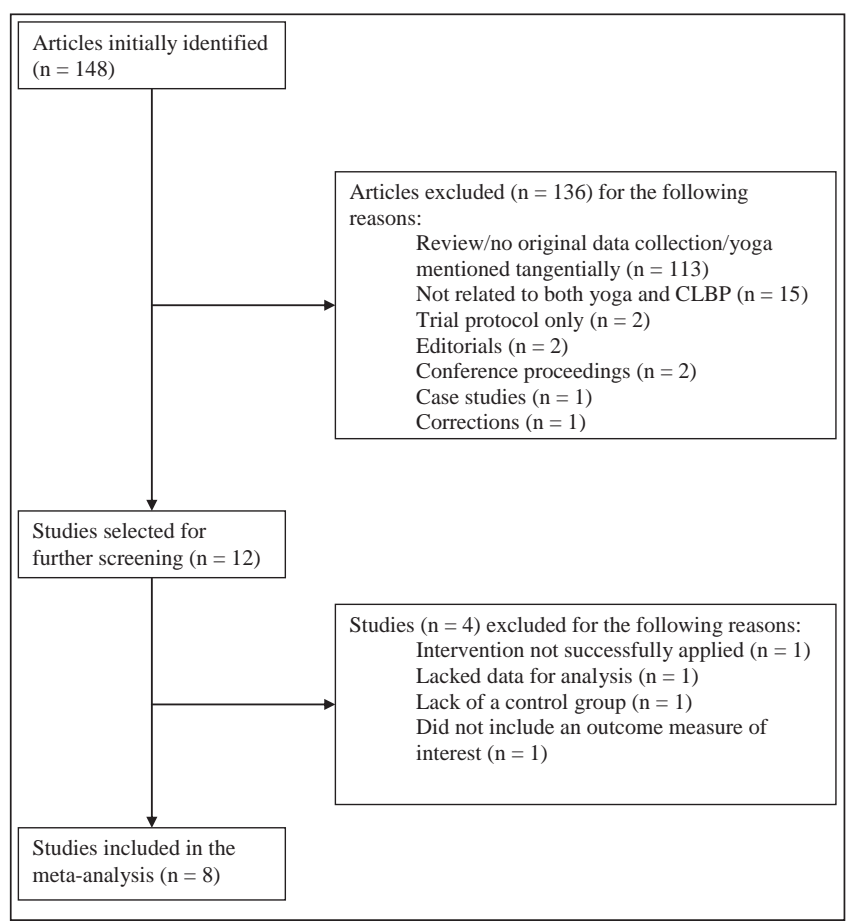

Figure 1) Study selection process. CLBP Chronic low back pain

significant reduction in pain intensity in five of these trials. Wren et al (18) reviewed six RCTs of yoga for CLBP and suggested that yoga shows promise in reducing pain, pain-related disability, negative mood and pain medication among patients with CLBP. Nonetheless, only two previous attempts have been made to quantify the effect sizes of yoga on CLBP across studies. The first meta-analysis included only two small RCTs (21). The second included six controlled trials of yoga for back pain (total $n=348$ ), but combined these results with yoga for other conditions, such as rheumatoid arthritis, and in healthy populations without chronic pain (22). Moreover, this review did not include the two most recent RCTs of yoga for $\operatorname{CLBP}(17,23)$, which are by far the largest $(n=228$ and $n=338)$ that have been conducted to date.

The goal of the present study was to conduct an updated meta-analysis of RCTs that have evaluated the efficacy of yoga specifically for CLBP, with a focus on pain and functional disability as treatment outcomes. In addition to evaluating the robustness of previous study findings regarding post-treatment effects and conducting a standardized assessment of study quality, the present study is, to our knowledge, the first to include a quantitative review of the follow-up treatment effects of yoga for CLBP. The present meta-analytical review and quality assessment was designed to guide future studies in this rapidly growing area of research and to provide clinically relevant information for patients and clinicians regarding the immediate and follow-up treatment effects of yoga for CLBP.

\section{METHOD}

Search procedure

The literature search strategy involved all relevant electronic databases available through the Electronic Research Databases (eg, Cumulative Index to Nursing and Allied Health Literature, Cochrane Central Register of Controlled Trials, Embase, Global Health, MEDLINE, PsycINFO, etc) at the University of British Columbia library (Vancouver, British Columbia). The primary search was comprehensive, seeking articles containing the words "Yoga" and "Back" anywhere in the text of peer-reviewed journals. Searches were also performed for "Yoga" and "CLBP", "Complementary and Alternative Medicine" and "CLBP," and "Yoga" and "Chronic Pain" as subject terms. The searches were performed between February 12, 2011 and
November 30, 2011, and were limited to articles that had their full body text written in English. Additional articles were sought by snowballing (checking the reference sections of the initial articles to identify further studies of interest). Authors who had published in this area were also contacted to determine whether they had any information regarding any unpublished research, but no additional manuscripts were identified.

\section{Inclusion criteria}

Studies needed to meet the following inclusion criteria to be included in the analysis:

- RCT;

- published in a peer-reviewed journal;

- full body text in English;

- yoga as a treatment for CLBP;

- only adult participants ( $\geq 18$ years of age);

- reported outcome measures of pain and/or functional disability;

- sufficient data reported to calculate effect sizes.

\section{Study selection}

A total of 148 articles were identified during the initial literature search and were considered for inclusion based on study relevance and inclusion criteria. The study selection process is detailed in Figure 1. No studies were excluded due to language restrictions. Of the 12 studies that were identified as potentially suitable for the present metaanalysis, four were excluded (23-26). One of the excluded studies was a feasibility trial designed to evaluate issues and challenges in conducting research on yoga as an intervention for CLBP (24). However, quantitative outcome data were not presented, and were not available from the study authors when contacted. Groessl et al (25) conducted a study of yoga for military veterans with CLBP, but their trial did not include a control group. An RCT by Tekur et al (26) evaluated the impact of yoga on quality of life and a straight-leg raise test, but not pain or functional disability. Finally, Cox et al's (27) pilot RCT of 20 participants was excluded due to significant concerns regarding treatment adherence. Specifically, one-half of the participants assigned to their yoga intervention did not attend any yoga classes, and the mean class attendance was 1.7 (of 12 possible classes).

\section{Quality assessment}

The eight included studies (17,23,28-33) were coded for quality by RTB and a trained independent rater using the checklist to evaluate a report of a nonparmacological trial (CLEAR NPT [34]). The CLEAR NPT was developed using a Delphi consensus method (based on the input of 55 experts, $68 \%$ of whom were members of the Cochrane collaboration) to assess the risk of bias in nonpharmacological trials. It addresses key components of RCT study quality (eg, participant attrition, blinding), as well as methodological issues unique to NPTs (eg, care provider experience or skill). The CLEAR NPT includes 10 items, as well as five subitems that take into consideration the fact that blinding of therapists and participants is often not feasible in NPTs, and is accompanied by a detailed user guide. The inter-rater reliability in the current study was good (kappa $=0.68)$, with agreement on $92 \%$ of the items. The most common source of disagreement between raters was regarding what qualifications were considered to be 'adequate' for the yoga instructors. Item discrepancies were resolved by consensus.

\section{Statistical analysis}

Statistical analyses were performed using SPSS version 17.0 (IBM Corporation, USA) and the meta-analysis macro available at $<$ www.spsstools.net/Syntax/MetaAnalysis $>$. Cohen's d was used as the estimate of the effect size (35). To take into consideration both group and baseline differences, parametric gain scores were used whenever possible for the calculation of Cohen's $d$. In general, d-based effect sizes can be categorized as small at the 0.20 level, medium at the 0.50 level and large at the 0.80 level (36). Due to the small number of studies included and the relative heterogeneity in study designs, a 


\begin{tabular}{|c|c|c|c|c|c|c|}
\hline Author (reference), year & $\mathbf{n}$ & Participant demographics & Treatment group (n) & Control group (n) & Pain outcome & $\begin{array}{c}\text { Functional } \\
\text { disability } \\
\text { outcome }\end{array}$ \\
\hline Galantino et al (28), 2004 & 16 & $\begin{array}{l}77 \% \text { female; } \\
\text { age range } 30-65 \text { years }\end{array}$ & $\begin{array}{l}\text { Hatha yoga; } \\
6 \text { weeks, twice per week (11) }\end{array}$ & No treatment (11) & Not assessed & ODI \\
\hline Sherman et al (29), 2005 & 66 & $\begin{array}{l}66 \% \text { female; } \\
\text { mean age } 44 \pm 13 \text { years }\end{array}$ & $\begin{array}{l}\text { Viniyoga; } \\
12 \text { weekly } 75 \text { min classes (36) }\end{array}$ & $\begin{array}{l}\text { Exercise (35); } \\
\text { self-care book (30) }\end{array}$ & $\begin{array}{l}\text { Bothersomeness of } \\
\text { pain (11-point NRS) }\end{array}$ & RDQ \\
\hline Williams et al (30), 2005 & 44 & $\begin{array}{l}68 \% \text { female; } \\
\text { mean age } 48.3 \pm 1.5 \text { years }\end{array}$ & $\begin{array}{l}\text { lyengar yoga; } \\
16 \text { weekly } 90 \text { min classes (20) }\end{array}$ & Education (24) & VAS & PDI \\
\hline Tekur et al (31), 2008 & 80 & $\begin{array}{l}46 \% \text { female; } \\
\text { mean age } 49 \pm 3.6 \text { years (yoga), } \\
48 \pm 4 \text { years (control) }\end{array}$ & $\begin{array}{l}\text { Hatha yoga and related practices; } \\
8 \text { h/day for } 7 \text { days (40) }\end{array}$ & Exercise (40) & $\begin{array}{l}\text { ODI (Section 1: pain } \\
\text { intensity) }\end{array}$ & ODI \\
\hline Williams et al (32), 2009 & 74 & $\begin{array}{l}77 \% \text { female; } \\
\text { mean age } 48 \text { years }\end{array}$ & $\begin{array}{l}\text { lyengar yoga; } \\
24 \text { weekly } 90 \text { min classes (31) }\end{array}$ & Waitlist (43) & $10 \mathrm{~cm}$ VAS & ODI \\
\hline Saper et al (33), 2009 & 30 & $\begin{array}{l}83 \% \text { female; } \\
\text { mean age } 44 \pm 12 \text { years }\end{array}$ & $\begin{array}{l}\text { Hatha yoga; } \\
12 \text { weekly } 75 \text { min classes (15) }\end{array}$ & Waitlist (15) & 11-point NRS & RDQ \\
\hline Sherman et al (23), 2011 & 228 & $\begin{array}{l}64 \% \text { female; } \\
\text { mean age } 48.4 \pm 9.8 \text { years }\end{array}$ & $\begin{array}{l}\text { Viniyoga; } \\
12 \text { weekly } 75 \text { min classes (92) }\end{array}$ & $\begin{array}{l}\text { Stretching (91); } \\
\text { self-care book (45) }\end{array}$ & $\begin{array}{l}\text { Bothersomeness of } \\
\text { pain (11-point scale) }\end{array}$ & RDQ \\
\hline Tilbrook et al (17), 2011 & 313 & $\begin{array}{l}68 \% \text { female (yoga)/73\% female } \\
\text { (control); } \\
\text { mean age } 46.4 \pm 11.3 \text { (yoga), } \\
46.3 \pm 11.5 \text { (control) }\end{array}$ & $\begin{array}{l}\text { Yoga; } \\
12 \text { weekly } 75 \text { min classes (156) }\end{array}$ & Usual care (157) & Not assessed & RDQ \\
\hline
\end{tabular}

NRS Numerical rating scale; ODI Oswestry Disability Index; PDI Pain Disability Index; RDS Roland-Morris Disability Questionnaire; VAS Visual analogue scale

random-effects model was used, using Dersimonian-Laird's method (37). Random-effects models do not presume that there is only one true effect size; rather, they allow for variance in methodologies and effect sizes between studies (38). Both post-treatment and follow-up effect sizes were calculated. For the post-treatment analyses, the earliest assessment of the outcome variables after treatment was used. For the follow-up analyses, the assessment closest to three months postintervention was used. Values from intention-to-treat analyses were used when available. Because methodological variance among studies can cause difficulties in the interpretation of the results of a meta-analysis, and because the majority of the RCTs included in the present meta-analysis used a waitlist or education control group, outcome data provided for the waitlist or education control groups were used when multiple control groups were included. Therefore, although Sherman et al $(23,29)$ used a self-care education and exercise/stretching control group in their trials, only the data from the self-care control group were included. For the same reason, results from the $10 \mathrm{~cm}$ visual analogue scale (VAS) in Williams et al (30) were used, rather than results from the present pain intensity scale (which assesses pain from 0 to 5 and was not used in any of the other studies). The VAS results provided a more conservative estimate of the effects found in Williams et al (30) compared with the present pain intensity results.

Orwin's (39) fail-safe $\mathrm{n}$ was calculated to determine the number of studies with an effect size of zero that would be required to reduce the effect size of yoga to 0.20 . The fail-safe $\mathrm{n}$ is derived using the following formula: $[\mathrm{n}(\mathrm{d}-\mathrm{dc})] / \mathrm{dc}$. In this formula, $\mathrm{n}$ is the number of studies in the meta-analysis, $\mathrm{d}$ is the mean effect size across the $\mathrm{n}$ studies, and $\mathrm{dc}$ is the criterion effect size to which d would drop (in this case, 0.20). Two statistical tests of the degree of heterogeneity were used: Cochrane's Q (based on the $\chi^{2}$ test) and the $I^{2}$ statistic $(40,41)$. Cochrane's $Q$ tests the null hypothesis that the included studies share a common effect size. The $\mathrm{I}^{2}$ statistic is an indication of the amount of variance among studies resulting from heterogeneity rather than chance. It represents heterogeneity as a percentage between 0 and 100 , with general guidelines indicating that $25 \%$ represents low, $50 \%$ represents moderate and $75 \%$ represents high heterogeneity (40). Due to the small number of studies included in the present metaanalysis, it was not possible to statistically examine sources of heterogeneity.

\section{RESULTS}

An overview of the study characteristics is provided in Table 1. Study participants were predominantly middle-age Caucasian women. Seven of the eight trials were conducted in the United States $(17,23,28$ $30,32,33)$ and one trial was conducted in India (31). Studies ranged in size from 16 (28) to 313 (17) participants. The styles of yoga included: Hatha ( $\mathrm{n}=3$; a general term denoting a focus on physical postures) $(28,31,33)$; Iyengar $(n=2$; a style of Hatha yoga that focuses on precision and alignment with extensive use of props) (30,32); Viniyoga ( $\mathrm{n}=2$; yoga involving an individually tailored sequences of poses) $(23,29)$; and an unspecified type $(n=1)(17)$. Control groups included in the analyses were education $(n=3)(29,30)$, exercise $(n=1)(31)$, waitlist control $(n=2)(32,33)$ and usual care $(n=2)(17,28)$. Six of the eight trials reported on session attendance, and attendance appeared to be quite high overall. In three trials (each consisting of 12 yoga sessions), the median session attendance was eight (33), nine (29) and 10 (23) sessions. In both of the trials by Williams et al $(30,32)$, participants attended approximately $90 \%$ of the yoga sessions. Another study reported that $60 \%$ of participants attended at least six of 12 yoga sessions (17). Regular home practice was encouraged across trials, but the recommended frequency and duration of home practice varied. Similarly, most studies assessed home practice, but adherence to homework log completion was inconsistent and adherence to home practice varied substantially within and across studies.

Pain and functional disability were assessed using scales that have generally demonstrated strong reliability and validity in past research. Pain was assessed in six trials, using either a VAS of pain intensity (42), a numerical rating scale of pain intensity (43), the pain intensity subscale of the Oswestry Disability Index (44) or a numerical rating scale of pain bothersomeness $(23,29)$. Measures of functional disability included the Oswestry Disability Index (44), the Roland-Morris Disability Scale (45) and the Pain Disability Index (46).

Summaries of the study findings, including individual and overall effect sizes, are presented in Table 2 (post-treatment results) and Table 3 (follow-up results). Of note, no serious adverse events were reported across studies. Minor adverse events (eg, increased pain) were rare. Of the 401 patients who were randomized to a yoga treatment group across the included studies, nine experienced an increase in pain that was "definitely" $(n=2)$, "probably or definitely" $(n=3)$, or "probably or possibly" $(n=4)$ attributable to yoga. 


\section{TABLE 2}

Post-treatment effect sizes of yoga for the treatment of chronic low back pain

\begin{tabular}{lccc}
\hline Author (reference), year & $\mathbf{n}$ & $\begin{array}{c}\text { Pain } \\
\text { (Cohen's d) }\end{array}$ & $\begin{array}{c}\text { Functional disability } \\
\text { (Cohen's d) }\end{array}$ \\
\hline Galantino et al (28), 2004 & 22 & $\mathrm{n} / \mathrm{a}$ & 0.49 \\
Sherman et al (29), 2005 & 66 & $\mathrm{n} / \mathrm{a}$ & 0.85 \\
Williams et al (30), 2005 & 44 & 0.38 & 0.40 \\
Tekur et al (31), 2008 & 80 & 0.27 & 1.01 \\
Williams et al (32), 2009 & 90 & 0.87 & 0.82 \\
Saper et al (33), 2009 & 30 & 0.90 & 0.34 \\
Sherman et al (23), 2011 & 137 & 0.74 & 0.62 \\
Tilbrook et al (17), 2011 & 274 & $\mathrm{n} / \mathrm{a}$ & 0.55 \\
Total n & & 381 & 743 \\
Cohen's d & & 0.623 & 0.645 \\
Orwin's fail-safe n & & 11 & 18 \\
\hline
\end{tabular}

n/a Not available

\section{Quality assessment}

The two most recent trials of yoga for CLBP satisfied all criteria outlined by the CLEAR NPT, with the exception of items regarding the blinding of therapists and participants (which was not feasible) $(17,23)$. Two of the eight trials included in the present meta-analysis failed to use an intention-to-treat analysis $(30,31)$. Three trials were unclear with respect to the concealment of treatment allocation $(28,30,32)$ and two trials were unclear regarding the use of skilled yoga therapists $(29,31)$. No RCT included in the present meta-analysis received a 'no' or 'unclear' on more than four of the possible 15 CLEAR NPT items and subitems. The most common weakness among the studies was the failure to adequately blind study participants and therapists, which was not feasible in the context of these yoga trials.

\section{Efficacy of yoga for pain}

The overall post-treatment effect size of yoga on pain was $d=0.623$ ( $95 \%$ CI 0.377 to $0.868 ; n=5$ ), indicating a medium to large effect size (36). Orwin's fail-safe $\mathrm{n}$ was 11 , indicating that 11 studies with nonsignificant results would have to have been missed for the treatment effect of yoga on pain to be reduced to a small effect size of 0.2 . The $\chi^{2}$ of heterogeneity test was nonsignificant $\left(\chi^{2}=5.16, \mathrm{df}=4 ; \mathrm{P}=0.27\right)$, and the $\mathrm{I}^{2}$ statistic was $22.4 \%$, indicating low variation in effect sizes among studies.

Compared with the post-treatment results, analysis of the follow-up data revealed a smaller effect size of $d=0.397$ (95\% CI 0.053 to 0.848; $\mathrm{n}=5)$. Orwin's fail-safe $\mathrm{n}$ was five. The degree of heterogeneity was significant $\left(\chi^{2}=15.86, d f=4 ; P=0.003\right)$, and the $I^{2}$ statistic was $74.8 \%$, indicating a high level of variability across studies.

\section{Efficacy of yoga for functional disability}

The overall post-treatment effect size of yoga on functional disability was $\mathrm{d}=0.645$ ( $95 \%$ CI 0.496 to $0.795 ; \mathrm{n}=8$ ), indicating a medium to large effect size (36). Orwin's fail-safe $\mathrm{n}$ was 18 . The $\chi^{2}$ of heterogeneity test was nonsignificant $\left(\chi^{2}=5.75, \mathrm{df}=7 ; \mathrm{P}=0.569\right)$, indicating there was no significant variation in the effect sizes among the included studies on this outcome measure. The $\mathrm{I}^{2}$ and Tau-square were both zero, indicating that a fixed-effect model would have yielded the same result for this analysis, and that there was limited variability among studies.

Analysis of the follow-up data for functional disability revealed a medium effect size of $d=0.486,(95 \%$ CI 0.226 to $0.746 ; n=6)$. Orwin's fail-safe $\mathrm{n}$ was nine. The heterogeneity of the included studies was higher than the post-treatment data, with a $\chi^{2}$ that was close to statistical significance $\left(\chi^{2}=10.67, \mathrm{df}=5 ; \mathrm{P}=0.058\right)$, and an $\mathrm{I}^{2}$ of $53.1 \%$ (in the moderate range).

\section{DISCUSSION}

Over the past several years, there has been a dramatic surge of interest among the Western scientific community regarding the possible
TABLE 3

Follow-up treatment effect sizes of yoga for the treatment of chronic low back pain

\begin{tabular}{|c|c|c|c|c|}
\hline Author (reference), year & $\mathbf{n}$ & $\begin{array}{l}\text { Follow-up } \\
\text { (number of } \\
\text { weeks post- } \\
\text { treatment) }\end{array}$ & $\begin{array}{c}\text { Pain } \\
\text { (Cohen's d) }\end{array}$ & $\begin{array}{l}\text { Functional } \\
\text { disability } \\
\text { (Cohen's d) }\end{array}$ \\
\hline Sherman et al (29), 2005 & 66 & 14 & 1.16 & 0.90 \\
\hline Williams et al (30), 2005 & 42 & 12 & 0.48 & 0.35 \\
\hline Williams et al (32), 2009 & 90 & 24 & 0.72 & 0.81 \\
\hline Saper et al (33), 2009 & 30 & 14 & -0.84 & -0.37 \\
\hline Sherman et al (23), 2011 & 137 & 14 & 0.42 & 0.48 \\
\hline Tilbrook et al (17), 2011 & 272 & 24 & $\mathrm{n} / \mathrm{a}$ & 0.41 \\
\hline Total $n$ & & & 365 & 637 \\
\hline Cohen's d & & & 0.397 & 0.486 \\
\hline Orwin's fail-safe $n$ & & & 5 & 9 \\
\hline
\end{tabular}

n/a Not available

health benefits of yoga for chronic pain, with a particular interest in its role in treating CLBP. To evaluate the efficacy of yoga as a treatment for CLBP, the present meta-analysis examined the post-treatment and follow-up results of eight RCTs that included a total of 743 patients. By limiting our review to only RCTs, our results provide a reliable, yet conservative, estimate of the efficacy of yoga for CLBP. The posttreatment effect sizes of yoga for pain and functional disability were both in the medium-to-large range $(d=0.623$ and $d=0.645$, respectively). At follow-up, effects were smaller, but still significant, with effect sizes in the small to medium range for pain and disability $(\mathrm{d}=0.397$ and $\mathrm{d}=0.486$, respectively). These findings are particularly encouraging given that CLBP is a highly prevalent and disabling condition that has historically been difficult to treat (46).

The results of our meta-analysis are also consistent with the moderate post-treatment effect sizes found in a recent meta-analysis by Bussing et al (22). However, in contrast to the moderate degree of heterogeneity identified by Bussing et al (22), we found low heterogeneity in the effect sizes for pain and disability. This difference is likely largely due to the fact that our analyses focused only on CLBP patients, and only on RCTs. Given that these differences (combined with a more than double-sized sample and two additional high-quality studies) led to lower effect-size variability in our study, we can further increase our confidence that yoga may represent an efficacious adjunctive treatment for CLBP. Compared with the results of metaanalyses of other popular nonpharmacological interventions for CLBP (47), we can also state that the effect size for yoga in reducing pain and functional disability appears to be similar to, if not higher than, effects sizes for more traditional exercise therapy (48), cognitive behavioural therapy (49) and acupuncture (50).

Overall, our findings provide the strongest support for the effects of yoga on short-term improvements in functional disability among patients with CLBP. Based on a total of eight RCTs, the moderate posttreatment effect size was a robust finding, with a fail-safe $\mathrm{n}$ of 18 and no significant heterogeneity across studies. It is interesting to note that a range of different yoga interventions yielded statistically similar effect sizes. Of the eight RCTs, three reported using Hatha yoga, two used Iyengar yoga, two used Viniyoga and one used an unspecified type of yoga. This suggests that yoga's general emphasis on strength, flexibility, breathing and focused awareness may be more important than the specific sequence or type of yoga postures ('asanas') used in different styles of yoga. However, the fact that one-half of the RCTs used a waitlist control group raises two important issues. First, without an active treatment control, one cannot rule out the possibility that the benefits of yoga were due (at least in part) to nonspecific treatment effects, such as extra attention, teacher enthusiasm and positive treatment expectations. Second, treatment gains may be attributable to the already established general benefits of physical activity for CLBP, and not specifically 
due to the effects of yoga $(7,48)$. Although Tekur et al (31) found yoga to be superior to "physical movements" and nonyogic breathing exercises on both pain and disability outcomes, they did not indicate the specific nature of these physical movements. Sherman et al's (29) first RCT of yoga for CLBP found that yoga led to statistically greater posttreatment improvements in functional status compared with a traditional exercise group (involving stretching, aerobic and strengthening exercises). However, this effect was not clinically significant and the effect dropped to a trend level of statistical significance by 14 weeks postintervention. Sherman et al's (23) more recent RCT compared yoga with stretching (and a small number of strengthening exercises) and failed to detect any significant differences in pain bothersomeness or disability between yoga and stretching groups at any timepoint. These mixed findings suggest a need for further research that compares the efficacy of yoga with other forms of physical activity, including traditional exercise programs and alternative therapeutic approaches (eg, pilates, tai chi).

The present study was, to our knowledge, the first to quantify the follow-up treatment effects of yoga for CLBP across previous RCTs. Based on the six RCTs that provided follow-up data (at three or six months), evidence for the longer-term benefits of yoga on pain and disability is promising, but the magnitude of treatment gains appears to be smaller and more heterogeneous than the post-treatment effects. Five of six RCTs demonstrated that treatment gains can persist for several months beyond the formal intervention period. In contrast, one RCT found that pain and disability actually worsened in the yoga group compared with the waitlist control group during the follow-up period (31). This was attributed to the fact that more than one-half of the yoga group (eight of the 15 participants) was lost to follow-up and one-third of the control group began practicing yoga during the follow-up period. Across all six studies, the more modest and variable effects sizes in the follow-up data could be related to the quantity and quality of yoga that patients practiced postintervention. Post-treatment adherence data presented in these studies suggests that participants varied in the extent to which they maintained a regular yoga practice beyond the formal intervention period. This is not surprising given that the study protocols themselves varied in the 'prescribed' amount of yoga practice, both during the intervention and postintervention. Further research is needed to determine the necessary and sufficient dose of yoga required during and after the treatment phase, and to better understand and address barriers to continued practice.

A number of other issues warrant attention in future research. For instance, the quality of yoga practice among patients with CLBP has received little attention. Although trials typically assess the quantity of yoga practiced (eg, number of minutes) during the intervention phase, they do not appear to assess whether patients are engaging postures correctly, with proper breath and focused attention to sensation. All of these components are presumed to be necessary to obtain the full benefits of a yoga practice (51). However, none of the eight RCTs conducted a statistical test of mediating factors in treatment outcomes, and the mechanisms through which yoga may improve patient well-being in CLBP remain poorly understood. Emerging research suggests that yoga likely improves patient pain and disability via a complex interaction of physiological, psychological and emotional pathways. For example, it has been proposed that yoga confers the physical benefits of increased core strength and stabilization, which are essential for back health (48). The fear-avoidance model of chronic musculoskeletal pain may also inform how yoga improves well-being in CLBP (52). According to this model, patients with low back pain may get trapped in a vicious cycle whereby threatening or catastrophic interpretations of pain and fear of pain lead to pain hypervigilance and avoidance of potentially pain-inducing activities. Over time, this hypervigilance and avoidance can lead to depression, disability and inactivity, which lead to further increases in pain, catastrophizing, etc. Yoga may help patients break out of this cycle by serving as a form of gradual, guided exposure to movement and activity. Indeed, a recent qualitative study of yoga suggested that yoga may help patients respond to pain and stiffness with movement, rather than rest and avoidance of activity (53). Across various chronic pain conditions, yoga has also been shown to reduce pain catastrophizing, increase pain self-efficacy, and decrease depression and anxiety $(14,15)$. However, an improvement in these factors has not always been found $(17,32)$.

Several limitations of the present meta-analysis need to be addressed. First, due to the relatively small number of RCTs that have evaluated the efficacy of yoga for CLBP, it was necessary to include studies that used a variety of yoga styles and varied substantially in intensity and duration. As a result, we were unable to draw conclusions regarding which type of yoga or intensity of yoga may be best for CLBP. Given the small number of RCTs (particularly those with follow-up data), we were also unable to conduct moderator analyses to determine whether effect sizes varied systematically based on factors such as study quality and adherence rates. Nonetheless, study quality appeared to be quite high across studies. Second, because we chose to use outcome data from waitlist and education control groups (rather than data from the stretching/exercise groups in the two studies by Sherman et al [23, 29]), our results cannot be generalized to the effects of yoga relative to other forms of physical activity. However, it is important to note that yoga did not perform worse than exercise in Sherman et al's trials. Together with the growing popularity of yoga among the general public, this suggests that yoga may be a viable alternative to more traditional exercise programs among patients who are more intrinsically motivated to participate in yoga. This is important given that intrinsic motivation has been linked to exercise adoption and maintenance (54).

CLBP is a highly prevalent and disabling medical condition associated with widespread emotional, physical and economic costs $(1,2)$. Despite the complex and heterogeneous etiology of CLBP, our metaanalysis suggests that yoga can have a medium-to-large-size effect on pain and functional disability, and that adverse effects are extremely rare when conducted under the supervision of a well-trained therapist. Our findings support and strengthen current recommendations for the use of nonpharmacological treatments among low back pain patients who do not improve with self-care approaches (7). As research on yoga for chronic pain progresses, it will be important for particular attention to be devoted to the underlying mechanisms of action of yoga and moderators of treatment effects. Perhaps most importantly, there is a need for high-quality RCTs that compare yoga with other active treatments, including cognitive behaviour therapy and other forms of exercise therapy, to determine whether yoga has specific effects. In doing so, researchers will also be better able to blind study participants and therapists to the 'active' treatment group, thereby reducing demand characteristics and equilibrating treatment expectations across study groups (a common weakness in past research). In summary, our findings suggest there is much promise, yet much to be learned, about yoga as a recommended therapeutic approach for those with CLBP.

ACKNOWLEDGEMENTS: The authors extend their sincere thanks to Dr Brian O'Connor for his valuable feedback regarding the statistical analyses, and to Hilla Shlomi for her assistance in the quality coding of RCTs.

\section{REFERENCES}

1. Lim KL, Jacobs P, Klarenbach S. A population-based analysis of healthcare utilization of persons with back disorders: Results from the Canadian community health survey 2000-2001. Spine 2006;31:212-8.

2. Woolf AD, Pfleger B. Burden of major musculoskeletal conditions. Bull World of Health Organ 2003;81:646-56.

3. Andersson GBJ. Epidemiological features of chronic low-back pain. Lancet 1999;354:581-5.

4. Balague F, Mannion AF, Fellise F, Cedraschi C. Non-specific low back pain. Lancet 2012;379:482-91.

5. Linton SJ. A review of psychological risk factors in back and neck pain. Spine 2000;25:1148-56.

6. Haldeman S, Dagenais S. A supermarket approach to the evidenceinformed management of chronic low back pain. Spine J 2008;8:1-7. 
7. Chou R, Qaseem A, Snow V, et al. Diagnosis and treatment of low back pain: A joint clinical practice guideline from the American College of Physicians and the American Pain Society. Ann Intern Med 2007;147:478-91.

8. Foltz V, St Pierre Y, Rozenberg S, et al. Use of complementary and alternative therapies by patients with self-reported chronic pain back: A nation-wide survey in Canada. Joint Bone Spine 2005;72:571-7.

9. Carneiro KA, Rittenberg JD. The role of exercise and alternative treatments for low back pain. Phys Med Rehabil Clin North Am 2010;21:777-92.

10. Gatchel RJ, Peng YB, Peters ML, Fuchs PN, Turk DC. The biopsychosocial approach to chronic pain: Scientific advances and future directions. Psychol Bull 2007;133:581-624.

11. Field T. Yoga clinical research review. Complement Ther Clin Pract 2011;17:1-8.

12. Ryba TV, Kaltenborn JM. The benefits of yoga for athletes: The body. Athletic Therapy Today 2006;11:32-34.

13. Roland KP, Jakobi JM, Jones GR. Does yoga engender fitness in older adults? A critical review. J Aging Phys Act 2011;19:62-79.

14. Carson JW, Carson KM. Jones KD, et al. A pilot randomized controlled trial of the Yoga of Awareness program in the management of fibromyalgia. Pain 2010;151:530-9.

15. Curtis K, Osadchuk A, Katz J. An eight-week yoga intervention is associated with improvements in pain, psychological functioning and mindfulness, and changes in cortisol levels in women with fibromyalgia. J Pain Res 2011;4:189-201.

16. John PJ, Sharma N, Sharma CM, Kankane A. Effectiveness of yoga therapy in the treatment of migraine without aura: A randomized controlled trial. Headache 2007;47:654-61.

17. Tilbrook HE, Cox H, Hewitt CE, et al. Yoga for chronic low back pain. Ann Intern Med 2011;155:569-78.

18. Wren AA, Wright MA, Carson JW, Keefe FJ. Yoga for persistent pain: New findings and directions for an ancient practice. Pain 2011;152:477-80.

19. Guzman J, Esmail R, Karjalainen K, et al. Multidisciplinary bio-psycho-social rehabilitation for chronic low back pain. Cochrane Database Syst Rev 2002(1):CD000963.

20. Posadzki P, Ernst E, Terry R, Lee MS. Is yoga effective for pain? A systematic review of randomized clinical trials. Complement Ther Med 2011;19:281-7.

21. Slade SC, Keating JL. Unloaded movement facilitation exercise compared to no exercise or alternative therapy on outcomes for people with nonspecific chronic low back pain: A systematic review. J Manipulative Physiol Ther 2007;30:301-311.

22. Bussing A, Ostermann T, Ludtke R, Michalsen A. Effects of yoga interventions on pain and pain-associated disability: A meta-analysis. J Pain 2012;1:1-9.

23. Sherman KJ, Cherkin DC, Wellman RD, et al. A randomized trial comparing yoga, stretching, and a self-care book for chronic low back pain. Arch Intern Med 2011;171:2019-26.

24. Jacobs BP, Mehling W, Avins AL, et al. Feasibility of conducting a clinical trial on hatha yoga for chronic low back pain: Methodological lessons. Altern Ther Health Med 2004;10:80-3.

25. Groessl EJ, Weingart KR, Aschbacher K, Pada L, Baxi S. Yoga for veterans with chronic low-back pain. J Altern Complem Med 2008;14:1123-9.

26. Tekur P, Chametcha S, Hongasandra RN, Raghuram N. Effect of yoga on quality of life of CLBP patients: A randomized control study. Int J Yoga 2010;3:10-7.

27. Cox H, Tilbrook H, Aplin J, et al. A randomised controlled trial of yoga for the treatment of chronic low back pain: Results of a pilot study. Complement Ther Clin Pract 2010;16:187-93.

28. Galantino ML, Bzdewka TM, Eissler-Russo J, et al. The impact of modified hatha yoga on chronic low back pain: A pilot study. Altern Ther Health Med 2004;10:56-9.

29. Sherman KJ, Cherkin DC, Erro J, Miglioretti DL, Deyo RA. Comparing yoga, exercise, and a self-care book for chronic low back pain: A randomized, controlled trial. Ann Intern Med 2005;143:849.
30. Williams KA, Petronis J, Smith D, et al. Effect of Iyengar yoga therapy for chronic low back pain. Pain 2005;115:107-17.

31. Tekur P, Singphow C, Nagendra HR, Raghuram N. Effect of shortterm intensive yoga program on pain, functional disability and spinal flexibility in chronic low back pain: A randomized control study. J Altern Complem Med 2008;14:637-44.

32. Williams K, Abildso C, Steinberg L, et al. Evaluation of the effectiveness and efficacy of Iyengar yoga therapy on chronic low back pain. Spine 2009;34:2066-76.

33. Saper RB, Sherman KJ, Cullum-Dugan D, Davis RB, Phillips RS, Culpepper L. Yoga for chronic low back pain in a predominantly minority population: A pilot randomized controlled trial. Altern Ther Health Med 2009;15:18-27.

34. Boutron I, Moher D, Tugwell P, et al. A checklist to evaluate a report of a nonpharmacological trial (CLEAR NPT) was developed using consensus. J Clin Epidemiol 2005;58:1233-40.

35. Vacha-Haase T, Thompson B. How to estimate and interpret various effect sizes. J Couns Psychol 2004;51:473-81.

36. Cohen J. A power primer. Psychol Bull 1992;112:155-9.

37. DerSimonian R, Laird N. Meta-analysis in clinical trials. Control Clin Trials 1986;7:177-88.

38. Borenstein A. Comprehensive meta-analysis by Biostat. 1997. <www.meta-analysis.com> (Accessed June 1, 2011).

39. Orwin RG. A fail-safe N for effect size in meta-analysis. J Educ Stat 1983;8:157-9.

40. Higgins JPT, Thompson SG, Deeks JJ, Altman DG. Measuring inconsistency in meta-analyses. Brit Med J 2003;327:557-60.

41. Gavaghan DJ, Moore AR, McQay HJ. An evaluation of homogeneity tests in meta-analysis in pain using simulations of patient data. Pain 2000;85:415-24.

42. Price DD, McGrath PA, Rafii A, Buckingham B. The validation of visual analogue scales as ratio scale measures for chronic and experimental pain. Pain 1983;17:45-56

43. Jensen MP, Karoly P, Braver S. The measurement of clinical pain intensity: A comparison of six methods. Pain 1986;27:117-26.

44. Fairbank JC, Couper J, Davies JB, O'Brien JP. The Oswestry low back pain disability questionnaire. Physiotherapy 1980;66:271-3.

45. Roland M, Morris R. A study of the natural history of back pain. Part I: Development of a reliable and sensitive measure of disability in low-back pain. Spine 1983;8:141-4.

46. Tait RC, Chibnall JT, Krause S. The pain disability index: Psychometric properties. Pain 1990;40:171-82.

47. Chou R, Huffman LH. Nonpharmacologic therapies for acute and chronic low back pain: A review of the evidence for an American Pain Society/American College of Physicians clinical practice guideline. Ann Intern Med 2007;147:492 (erratum in Ann Intern Med 2008;148:247).

48. Hayden JA, van Tulder MW, Malmivaara AV, Koes BW. Meta-analysis: Exercise therapy for nonspecific low back pain. Ann Intern Med 2005;142:765-75.

49. Hoffman BM, Papas RK, Chatkoff DK, Kerns RD. Meta-analysis of psychological interventions for chronic low back pain. Health Psychol 2007;26:1-9.

50. Manheimer E, White A, Berman B, Forys K, Ernst D. Meta-analysis: Acupuncture for low back pain. Ann Intern Med 2005;142:651-63

51. Woodyard C. Exploring the therapeutic effects of yoga and its ability to increase quality of life. Int J Yoga 2011;4:49-54.

52. Vlaeyen JW, Linton SJ. Fear-avoidance and its consequences in chronic musculoskeletal pain: A state of the art. Pain 2000;85:317-32.

53. Tul R, Unruh A, Dick BD. Yoga for chronic pain management. Scand J Caring Sci 2010;25:435-43.

54. Buckworth J, Lee RE, Regan G, Schneider LK, DiClemente CC. Decomposing intrinsic and extrinsic motivation for exercise: Application to stages of motivational readiness. Psych Sport Exerc 2007;8:441-61. 


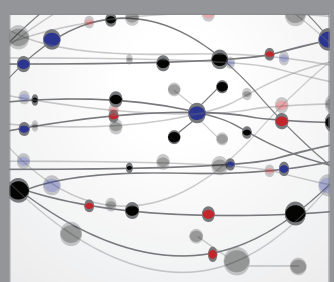

The Scientific World Journal
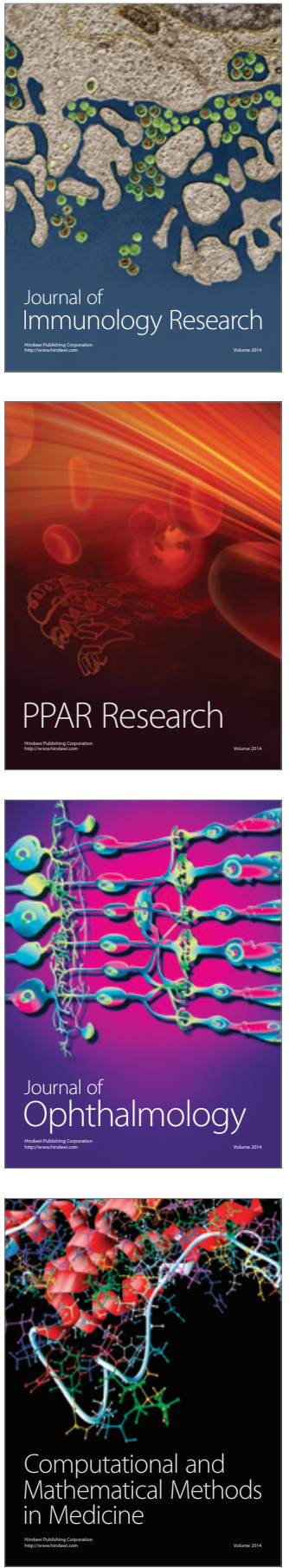

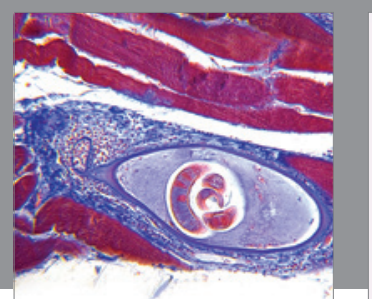

Gastroenterology Research and Practice

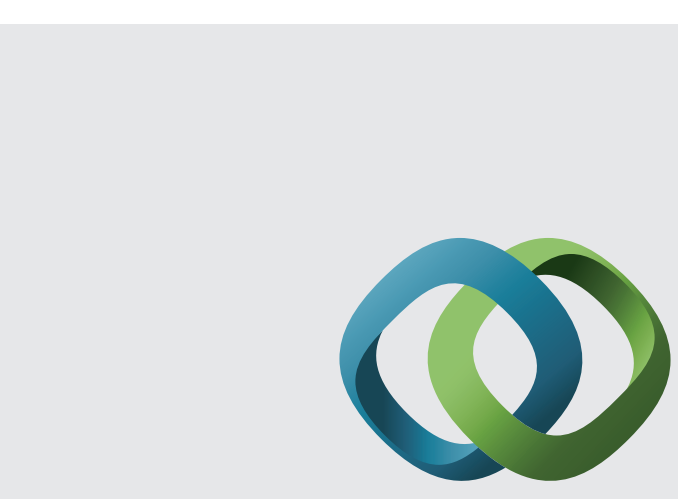

\section{Hindawi}

Submit your manuscripts at

http://www.hindawi.com
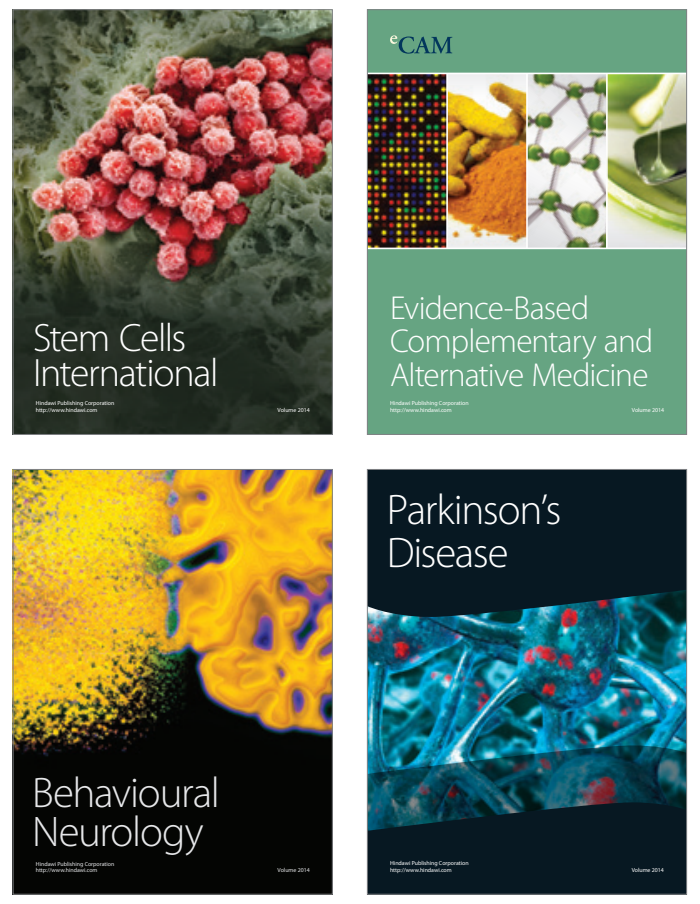
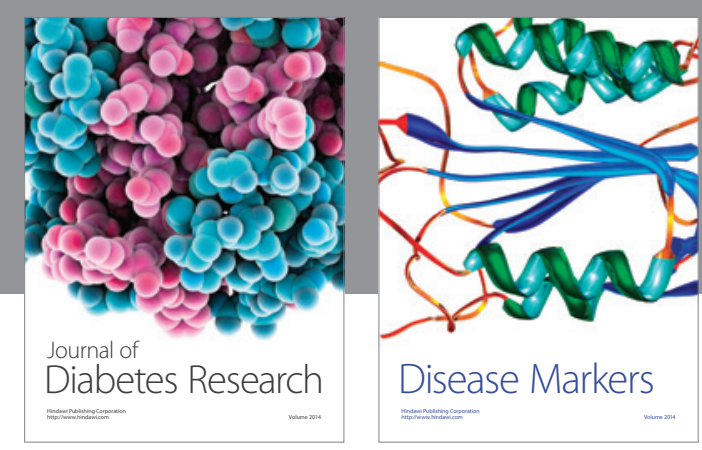

Disease Markers
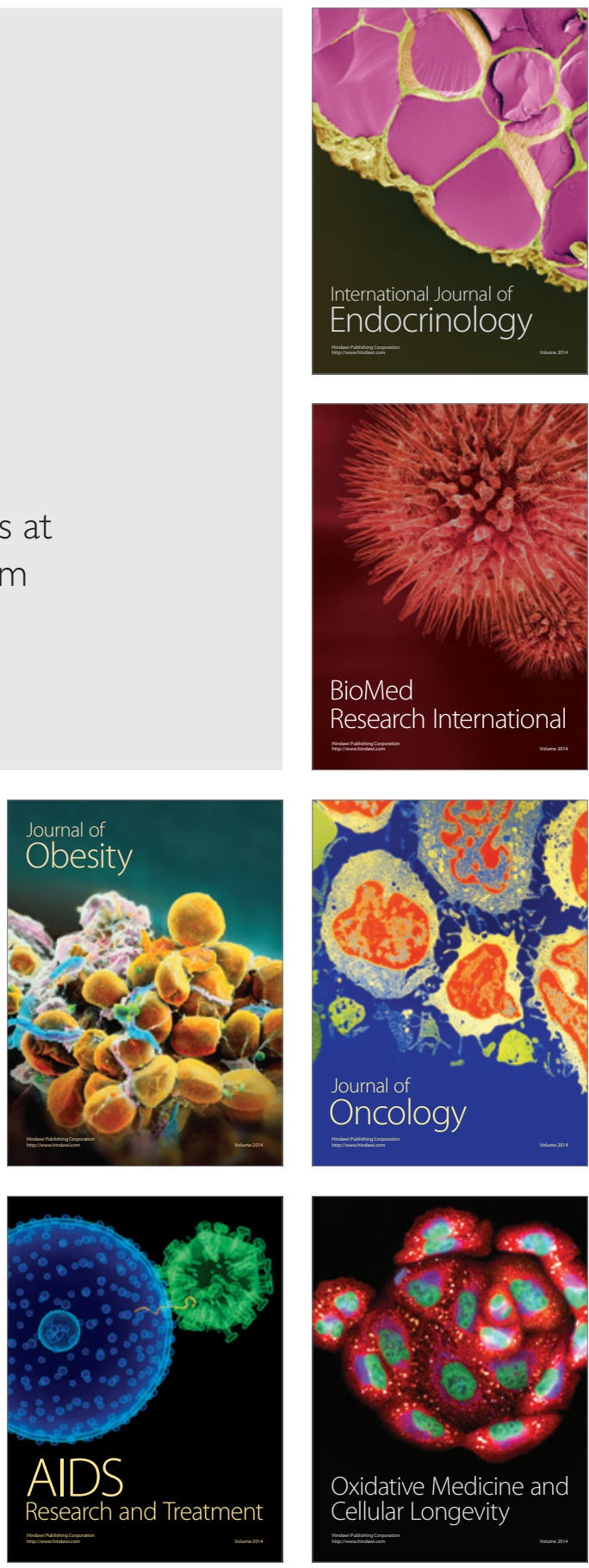